\title{
Marketing personal y empleo de los graduados de la Escuela Profesional de Administración, UNASAM, 2014
}

\author{
Personal Marketing and employment of Professional graduates of the School \\ of Administration, UNASAM, 2014
}

Telmo Loli Poma ${ }^{1}$, Félix Lirio Loli ${ }^{1}$, Juan Vílchez Cárcamo ${ }^{1}$ e Isaac Morales Cerna ${ }^{1}$

\section{RESUMEN}

El objetivo de la investigación fue analizar la influencia del marketing personal en el empleo de los graduados de la Escuela Profesional de Administración de la Universidad Nacional Santiago Antúnez de Mayolo. La investigación es de carácter aplicativo y el diseño usado para analizar la certeza de la hipótesis en el contexto particular que se plantea en la investigación fue no experimental, transversal y correlacional. La información fue recogida a través de una encuesta aplicada a los egresados con Título Profesional de Licenciado en Administración de la Universidad Nacional Santiago Antúnez de Mayolo, año 2011. Se concluye que el marketing personal influye significativamente en el empleo de los graduados, debido a que los titulados que no acuden a cursos de formación sobre búsqueda de empleo o de orientación profesional, presentan una tendencia hacia valoraciones de insatisfacción con el empleo en general respecto a su primer trabajo.

Palabras clave: marketing personal; empleo; graduados.

\begin{abstract}
The main objective of the research was to analyze the influence of personal marketing on the employment of Management Professional School graduates of the Santiago Antúnez de Mayolo National University. The research is of an application nature and the design used to analyze the certainty of the hypothesis in the particular context that arises in the research was non-experimental, transversal and correlational. The
\end{abstract}

1 Universidad Nacional Santiago Antúnez de Mayolo. Huaraz, Perú. 
information was collected through a survey applied to graduates with a Professional Degree from the Santiago Antúnez de Mayolo National University in 2011. It is concluded that personal marketing significantly influences the employment of the graduates, because Graduates who do not attend training courses on job search or vocational guidance have a tendency towards assessments of dissatisfaction with employment in general regarding their first job.

Keywords: personal marketing; job; graduates.

\section{ICHIKLLACHAW}

Kay musyapakuychawqa tarintsik imanawmi kikin markitin nishqan UNASAMchaw yachakuyushashqakuna uryata tariyanqantawanmanatariyanqantam. Kaymusyapakuyqa tsay llapantam tsuyantsaatsimun. Kay musyapakuyqa mana ikpirimintal nishqantam wanashqa, hinaman transwirsalwan kurilasiyunalwanmi rurakashqa. Tsaychaw llapan willakuyqa tapukuykunawanmi ayllukashqa, UNASAMpiq 2011chaw llapan yachakuqkuna yarqushqakunawannam. Kaychaw riqitsimantsik kay markitin nishqan alli yarqunqantam, alli tinkunqantam llapan ushashqa yachakuq rurayninkunawan, yarquyninkunallachaw mana urya ashiqkunaqa mana alli hina llakishqam kayan nimantsikmi.

Pushaq shimikuna: kikin markitin nishqan; urya; yachakuyninkunata ushaqkuna.

\section{INTRODUCCIÓN}

El propósito de nuestra investigación es analizar la influencia del marketing personal en el empleo de los graduados de la Escuela Profesional de Administración de la Universidad Nacional Santiago Antúnez de Mayolo. El marketing personal se ha convertido en un tema muy importante en los últimos años. Según Alles (2012), la expresión «marketing personal» hace referencia a una serie de acciones planeadas que se realizan con el propósito de incrementar las posibilidades de conseguir un nuevo empleo (mejorar la empleabilidad). Las acciones a realizar abarcan diferentes planos, comenzando por el interno, como por ejemplo, realizar una reflexión profunda para conocer a fondo las propias posibilidades. Luego analizar los aspectos externos, comenzando por el análisis de las opciones disponibles. Al respecto, Acosta (2006) sostiene que actualmente para el profesional resulta más eficaz y, sobre todo, mucho más gratificante apoyar el éxito, los puntos fuertes propios y cubrir las propias necesidades profundas. Por encima de 
todo, el éxito de cada persona debe estar regido por su jerarquía de valores, y orientado a satisfacer.

El acceso al empleo y el proceso de marketing personal que desarrollan los jóvenes universitarios egresados de la carrera profesional de Administración en la Universidad Nacional Santiago Antúnez de Mayolo no está liberado de dificultades e incertidumbre. Así, se observa generalmente que el sector de absorción de los titulados universitarios es la empresa privada. Por otro lado, varios graduados al ser ya profesionales y no tener un empleo, toman decisiones a corto plazo para la supervivencia o la mera preparación de circunstancias más propicias para adquirir experiencia y enriquecer su currículum. Además, son sólo unos pocos los que desarrollan un trabajo directamente vinculado con sus estudios universitarios.

El periodo de prolongación de búsqueda de empleo es responsabilidad del egresado, ya que al ser estudiante, olvida un hecho importante: las bases de lo que será o no su futuro laboral se empiezan a construir durante su trayectoria formativ»; así la transición al mundo laboral no es un hecho puntual que tiene lugar cuando recibe el título. La transición se inicia en la Universidad, pues puede complementar los estudios con otros componentes formativos que ampliaron su currículum (idiomas, cursos, estancias en otras universidades), compaginar los estudios con un trabajo, a fin de adquirir formación práctica, pero también establecer una red de contactos que le permitirá obtener un trabajo, y aprovechar o no, las oportunidades extracurriculares (conferencias, talleres, asociación) que la vida universitaria le ofrece.

Según Caballero y Kington (2005), en relación a la red de contactos, existen tres ejes principales del capital social y se puede considerar un factor importante en la inserción laboral, partiendo de la cooperación, confianza que un individuo desarrolla en las redes sociales que posee, los cuales tienen mediación en la estructura social de las dinámicas del mercado laboral. Aquellos con quienes se tiene vínculos fuertes están más motivados para ayudar sobre información acerca de trabajo (Granovetter, 1973: 1371).

En el escenario donde coinciden las personas que buscan una ocupación y el empleador, las estrategias de la persona y las estrategias de reclutamiento, representan vías de ofrecimiento de trabajo y selección con criterios y factores de contratación por parte de las empresas que requieren de graduados; es, además, el escenario inmediato de las conductas descritas de inserción en el cual tienen lugar las variables como actitudes, aspiraciones y expectativas sobre el mercado de trabajo; entonces el nivel de aceptación de una ocupación o un puesto de trabajo por el graduado, se considera como dependiente de las preferencias individuales y la capacidad de resistencia. Además, los 
atributos sociales como la clase social y el sexo tienden a crear una serie de expectativas en relación al segmento del mercado de trabajo al que se incorpora el egresado y, en consecuencia, el tipo de recompensas y gratificaciones que trataría de obtener de un empleo.

Lo mencionado afecta a la sociedad en general y al estrato de graduados en Administración, sus externalidades recaen en la débil acumulación de experiencia laboral y escasos hábitos de trabajo, periodo de búsqueda de empleo más largos, genera una carga financiera familiar, incertidumbre, desmotivación profesional, aumento del sector informal, subempleo o mal aprovechamiento del capital humano, emigración o fuga de mentes brillantes, ineficiente calidad de los servicios en los sectores económicos.

A continuación, se presenta la metodología seguida para estimar la satisfacción de los egresados con el empleo en general y las calificaciones en el grado de acuerdo a los factores psicosociales, para finalmente evaluar si existe correlación entre el marketing personal y el empleo.

\section{MATERIALES Y MÉTODOS}

De acuerdo con la orientación, la investigación es de carácter aplicativo debido a que la investigación estuvo dirigida a utilizar el conocimiento para generar soluciones a problemas prácticos relacionados con el tema de empleo de los egresados de la Escuela Académico Profesional de Administración. El diseño usado para analizar la certeza de la hipótesis en el contexto particular que se plantea en la investigación fue no experimental, transversal y correlacional. Transversal, debido a que los datos se recopilaron en un solo momento; correlacional, porque permitió evaluar la relación entre el marketing personal y el empleo de los graduados de la Escuela Profesional de Administración de la Universidad Nacional Santiago Antúnez de Mayolo. La población estuvo conformada por los 38 egresados que optaron el Título Profesional de Licenciado en Administración en la Escuela Profesional de Administración de la Universidad Nacional Santiago Antúnez de Mayolo en el año 2011. La técnica de recolección de datos fue la encuesta con su instrumento, el cuestionario. Para su aplicación se tuvo en cuenta los aspectos éticos como el consentimiento de los encuestados y protección de su privacidad. Recopilados los dados, se midió inicialmente cada una de las variables mediante tablas de frecuencia y, para análisis de la información, es decir, para establecer la relación entre las variables, se empleó el coeficiente de correlación de Pearson. 


\section{RESULTADOS}

Tabla 1. Valoración del grado de satisfacción respecto a factores psicosociales

\begin{tabular}{|c|c|c|c|c|}
\hline \multirow{2}{*}{ Factores Psicosociales } & \multicolumn{4}{|c|}{$\begin{array}{l}\text { Valoración de los factores psicosociales } \\
\text { Ni de acuerdo }\end{array}$} \\
\hline & En desacuerdo & $\begin{array}{l}\text { ni en des- } \\
\text { acuerdo }\end{array}$ & De acuerdo & Total \\
\hline \multicolumn{5}{|l|}{ IMPORTANCIA QUE OTORGAN AL EMPLEO } \\
\hline \multirow[t]{2}{*}{$\begin{array}{l}\text { Pienso que trabajar es una manera de ser útil para la sociedad y } \\
\text { me facilita relacionarme y autorrealizarme. }\end{array}$} & 4 & 1 & & 5 \\
\hline & $80 \%$ & $20 \%$ & $0 \%$ & $100 \%$ \\
\hline \multirow{2}{*}{$\begin{array}{l}\text { ¿Prefiere trabajar y tener independencia económica antes que } \\
\text { vivir de su familia? }\end{array}$} & 3 & & 2 & 5 \\
\hline & $60 \%$ & $0 \%$ & $40 \%$ & $100 \%$ \\
\hline \multirow{3}{*}{$\begin{array}{l}\text { ATRIBUCIÓN DE LAS CAUSAS DEL EMPLEO } \\
\text { Una de las razones por la que no encuentro trabajo es por falta } \\
\text { de formación, de experiencia, de carácter, por no saber dónde } \\
\text { buscarlo. }\end{array}$} & & & & \\
\hline & 4 & & 1 & 5 \\
\hline & $80 \%$ & $0 \%$ & $20 \%$ & $100 \%$ \\
\hline \multirow[t]{2}{*}{ Dedico poco esfuerzo para buscar trabajo } & 3 & & 2 & 5 \\
\hline & $60 \%$ & $0 \%$ & $40 \%$ & $100 \%$ \\
\hline \multirow[t]{2}{*}{$\begin{array}{l}\text { DISPONIBILIDAD PARA EL EMPLEO } \\
\text { Estoy dispuesto/a bajar mi nivel de aspiraciones profesionales } \\
\text { con tal de trabajar. }\end{array}$} & 2 & 3 & & 5 \\
\hline & $40 \%$ & $60 \%$ & $0 \%$ & $100 \%$ \\
\hline \multirow{2}{*}{$\begin{array}{l}\text { Aceptaría un trabajo por el que tuviese que cambiar mi lugar } \\
\text { de residencia o aceptaría cualquier horario ante una oferta de } \\
\text { empleo. }\end{array}$} & 3 & 2 & & 5 \\
\hline & $60 \%$ & $40 \%$ & $0 \%$ & $100 \%$ \\
\hline \multirow[t]{2}{*}{ Podría asumir salarios bajos y responsabilidades altas. } & 2 & 3 & & 5 \\
\hline & $40 \%$ & $60 \%$ & $0 \%$ & $100 \%$ \\
\hline \multirow[t]{2}{*}{$\begin{array}{l}\text { AUTOVALORACIÓN PERSONAL Y PROFESIONAL } \\
\text { Soy muy constante y perseverante a la hora de conseguir algo } \\
\text { que me interesa. }\end{array}$} & 3 & & 2 & 5 \\
\hline & $60 \%$ & $0 \%$ & $40 \%$ & $100 \%$ \\
\hline \multirow{2}{*}{$\begin{array}{l}\text { Considero que mi arreglo personal es adecuado a las ocupacio- } \\
\text { nes a las que aspiro. }\end{array}$} & 2 & & 3 & 5 \\
\hline & $40 \%$ & $0 \%$ & $60 \%$ & $100 \%$ \\
\hline \multirow{3}{*}{$\begin{array}{l}\text { Me considero un/a buen/a profesional. } \\
\text { ESTILO DE BÚSQUEDA DE EMPLEO } \\
\text { Realizo una búsqueda de empleo planificada organizando mi } \\
\text { actuación. }\end{array}$} & 1 & 2 & 2 & 5 \\
\hline & 5 & & & 5 \\
\hline & $100 \%$ & $0 \%$ & $0 \%$ & $100 \%$ \\
\hline \multirow{2}{*}{$\begin{array}{l}\text { Mientras busco trabajo ayudo a mis amigos en alguna actividad } \\
\text { aunque no remunerada, estudio o mantengo contactos con } \\
\text { profesionales de mi ramo. }\end{array}$} & 3 & 2 & & 5 \\
\hline & $60 \%$ & $40 \%$ & $0 \%$ & $100 \%$ \\
\hline \multicolumn{5}{|l|}{ MADUREZ OCUPACIONAL } \\
\hline \multirow[t]{2}{*}{$\begin{array}{l}\text { Me preocupo de informarme sobre cómo evoluciona mi profe- } \\
\text { sión con las nuevas tecnologías. }\end{array}$} & 5 & & & 5 \\
\hline & $100 \%$ & $0 \%$ & $0 \%$ & $100 \%$ \\
\hline \multirow[t]{2}{*}{$\begin{array}{l}\text { Estaría dispuesto a trabajar en otras ocupaciones distintas a mi } \\
\text { profesión. }\end{array}$} & 1 & 4 & & 5 \\
\hline & $20 \%$ & $80 \%$ & $0 \%$ & $100 \%$ \\
\hline \multicolumn{5}{|l|}{ HABILIDADES PARA LA BÚSQUEDA DE EMPLEO } \\
\hline \multirow{3}{*}{$\begin{array}{l}\text { Con frecuencia envío cartas y currículum a empresas o me dirijo } \\
\text { personalmente a ellas. }\end{array}$} & $\begin{array}{c}3 \\
60 \%\end{array}$ & $\begin{array}{c}2 \\
40 \%\end{array}$ & $0 \%$ & $\begin{array}{c}5 \\
100 \%\end{array}$ \\
\hline & 4 & 1 & & 5 \\
\hline & $80 \%$ & $20 \%$ & $0 \%$ & $100 \%$ \\
\hline
\end{tabular}


Los egresados que optaron el título profesional de Licenciado en Administración en el año 2011, pero cuya situación laboral actual es inactivo (5 egresados) respondieron sobre cada uno de los factores psicosociales, los resultados fueron: 5 (100\%) manifestaron estar en desacuerdo con las afirmaciones «Realizo una búsqueda de empleo planificada organizando mi actuación» y «Me preocupo de informarme sobre cómo evoluciona mi profesión con las nuevas tecnologías»; 4 (80\%) indicaron estar en desacuerdo con las afirmaciones «Pienso que trabajar es una manera de ser útil para la sociedad y me facilita relacionarme y autorrealizarme», "Una de las razones por la que no encuentro trabajo es por falta de formación, de experiencia, de carácter, por no saber dónde buscarlo», y 3 (60\%) respondieron estar en desacuerdo con las afirmaciones «Prefieró trabajar y tener independencia económica», «Aceptaría un trabajo por el que tuviese que cambiar mi lugar de residencia», «Soy muy constante y perseverante», «Mientras busco trabajo ayudo a mis amigos en alguna actividad, estudio o mantengo contactos con profesionales de mi ramo», «Sé comportarme en una entrevista de selección».

Tabla 2. Importancia de la serie de factores y competencias para el desempeño laboral

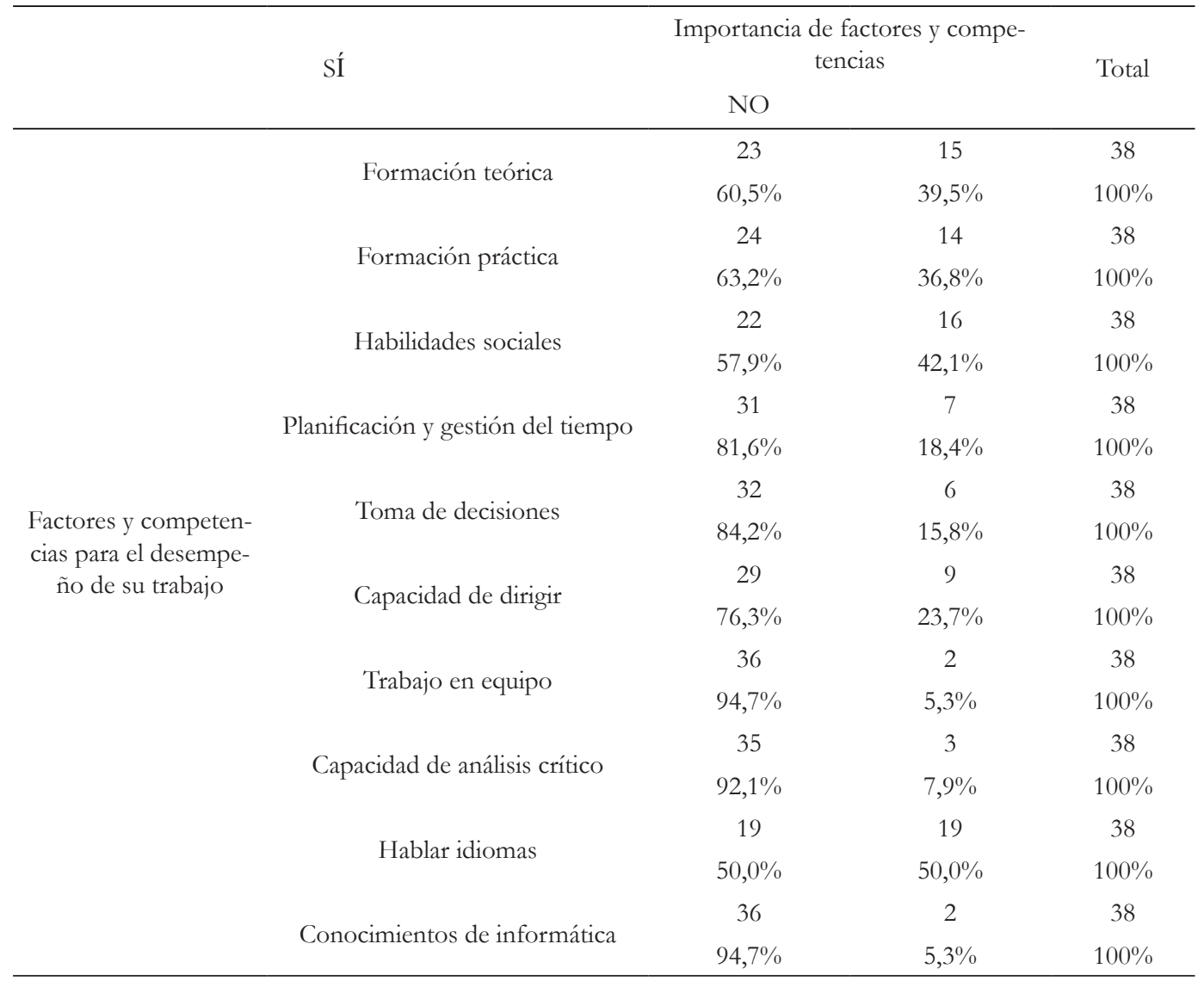


Al total de egresados que optaron el título profesional de Licenciado en Administración en el año 2011(38 egresados), se les preguntó sobre la importancia de cada uno de los factores y competencias para el desempeño laboral, los resultados fueron: el 23 $(60,5 \%)$ considera que el factor formación teórica sí es importante, 24 (63,2\%) respondió que la formación práctica es importante, 22 (57,9\%) indicó que sí en las habilidades sociales, $31(81,6 \%)$ en la planificación y gestión del tiempo, $32(84,2 \%)$ en el factor toma de decisiones, 29 (76,3\%) en el trabajo en equipo, 36 (94,7\%) respondieron que sí en el factor capacidad de análisis crítico, 35 (92,1\%) indicaron que hablar idiomas es importante y $36(94,7)$ considera que los conocimientos en informática sí son importantes para el desempeño laboral.

Contrastación de la hipótesis:

Tabla 3. Cursos de formación desarrollados sobre búsqueda de empleo VS Satisfacción con el empleo

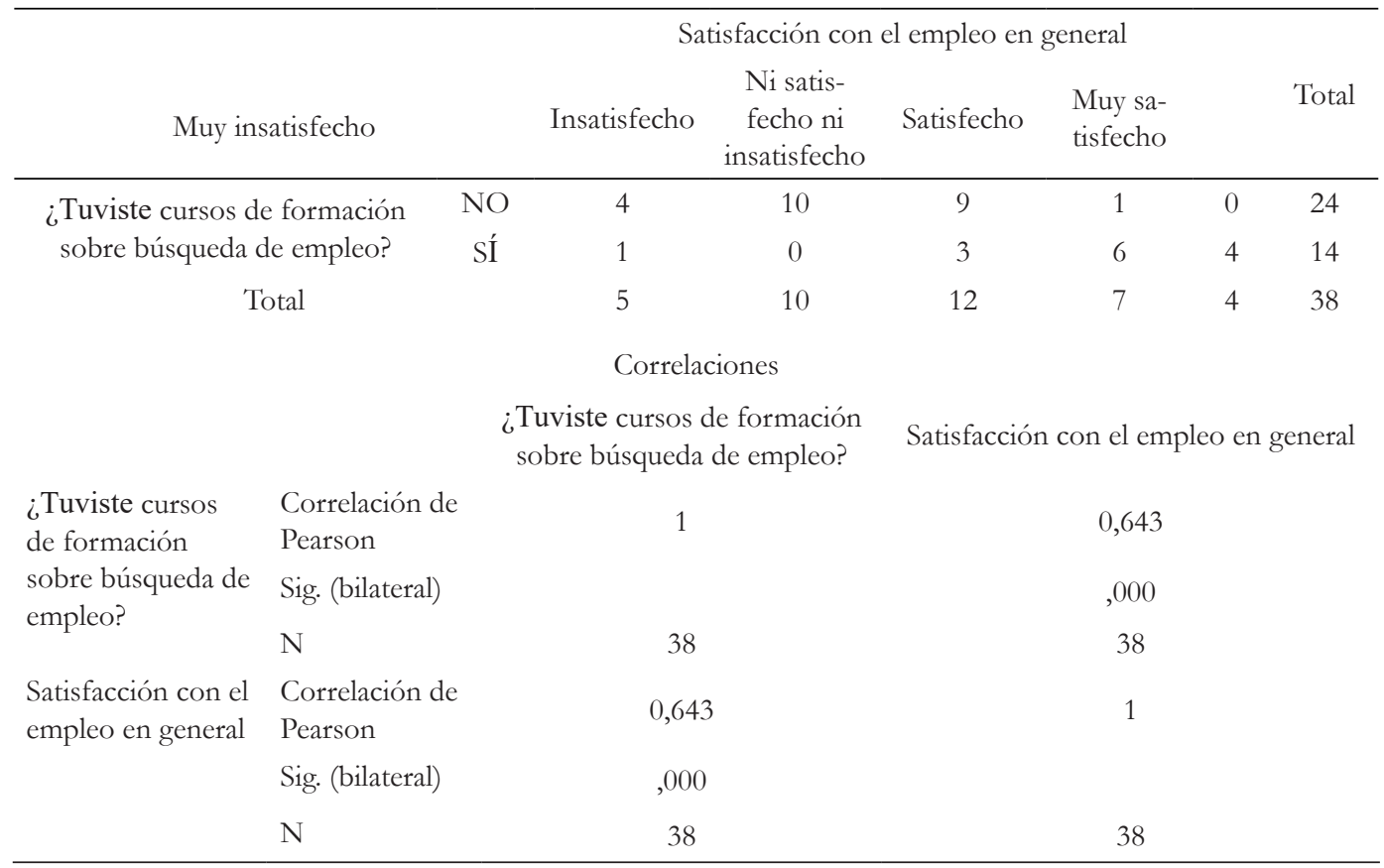

La correlación entre las puntuaciones de «tuviste cursos de formación sobre búsqueda de empleo y la satisfacción con el empleo en general», es el punto 0,643 con valor de $\mathrm{p}=0,000$ que, por lo tanto, es significativo $(0,000<0,05)$ y la correlación es buena $(0,643)$, lo que lleva a rechazar la hipótesis nula concluyendo que «el marketing personal influye significativamente en el empleo de los graduados de la Escuela de Administración de la UNASAM. 


\section{DISCUSIÓN}

La investigación alcanzó el objetivo principal, orientado a «analizar la influencia del marketing personal en el empleo de los graduados de la Escuela Profesional de Administración de la Universidad Nacional Santiago Antúnez de Mayolo». De acuerdo con los resultados se encontró que, en su mayoría, los titulados no concurren a cursos de formación sobre búsqueda de empleo o de orientación profesional, que son elementos importantes del marketing personal, lo cual genera una tendencia a la insatisfacción laboral con su primer empleo.

Al respecto, Fernández Guillén y otros (2001) señalan que la experiencia de la inserción laboral muestra que es preciso construir estructuras que faciliten el tránsito y el posterior acceso al mercado de trabajo. En unos casos, las estructuras de inserción posibilitan el aprendizaje de aptitudes y actitudes básicas, como se hace en los procesos de talleres sociales, en otros se desarrolla dicho aprendizaje mediante la participación en Centros de Inicio a la Actividad Laboral (Talleres de Inserción) o en las Empresas de Inserción. El conjunto de estas estructuras ofrece a las personas instrumentos para desarrollar y adquirir unas condiciones mínimas de empleabilidad. El segundo brazo del proceso de inserción laboral consiste en crear oportunidades de acceso a los mercados de trabajo, mediante el desarrollo de proyectos personalizados de búsqueda activa de empleo. El conocimiento de los requerimientos de perfiles profesionales que exigen las diferentes actividades y mercados de trabajo permite realizar una orientación inteligente de la búsqueda activa de empleo.

Este hecho de no contar con un marketing personal adecuado de los graduados que les permitan incrementar las posibilidades de conseguir un nuevo empleo y la existencia de una satisfacción laboral con su empleo, se hace más notorio a partir de los resultados que nos indican que la mayoría de los titulados no tardó más del tiempo de estipulado (5 años) en terminar su carrera; no obstante, la mayoría de titulados no se encuentra ni satisfecho(a) ni insatisfecho(a) con los estudios cursados. Lo mencionado coincide con los resultados de la investigación de Jiménez (2013), quien concluye que pueden aplicarse las tácticas de marketing personal como ventaja para ser más exitosos en el posicionamiento laboral; además, concuerda con los hallazgos de Sáenz (2013), que concluye que la formación técnica-empresarial se relaciona directamente con la inserción laboral de los egresados del Centro Técnico Productivo Teófilo Méndez Ramos de Huaraz; y, coincide también con los resultados de la investigación de Torres (2011) que, como conclusión identifica los elementos de un plan de marketing para el desarrollo de una marca personal. 
La experiencia acumulada por Cáritas, en el apoyo a los procesos de inserción laborales (durante más de 10 años), muestra cómo la búsqueda de empleo no es sólo cuestión de poseer información sobre las ofertas que puedan existir, ya que también existe un importante número de personas que, debido a las carencias que arrastran de tipo educativo, cultural, social y familiar, no poseen las condiciones mínimas de empleabilidad que se requiere para desempeñar cualquier trabajo (Cáritas Española, 1999: 8).

De modo más específico, se comprobó que, los factores psicosociales se relacionan directa y significativamente con el nivel de empleo de los graduados de la Escuela Profesional de Administración de la Universidad Nacional Santiago Antúnez de Mayolo al obtenerse como resultado puntuaciones bajas, valoradas para cada uno de los 16 elementos del total de siete factores psicosociales (importancia que otorgan al empleo, atribución de las causas del empleo, disponibilidad para el empleo, autovaloración personal y profesional, estilo de búsqueda de empleo, madurez ocupacional y habilidades para la búsqueda de empleo) por los titulados cuya situación laboral es la de inactivo(a). Hoy en día, las organizaciones laborales en general, y el mercado laboral de los graduados en particular, dan mucha importancia el perfil del candidato. De este perfil, destaca el saber hacer, o sea, el bagaje de conocimientos y destrezas y la capacidad para aplicar este bagaje en un contexto real. Pero de manera creciente se da valor, además, a las características personales, al saber ser, que incluye aspectos como las actitudes, la motivación y el compromiso con el trabajo o la capacidad de adaptación, cualidades que son reconocidas con creces como básicas para la productividad laboral. La dificultad y/o el coste económico que supone valorar estas características se suplen mediante las redes de conocidos, el aval de los cuales permite simplificar el proceso de reclutamiento y selección. A menudo, con el mundo universitario, suele ser recurso para la búsqueda de estas personas; sin embargo hay otros, como el valor de las prácticas.

Asimismo se demostró que la experiencia pre-profesional se relaciona directa y significativamente con el empleo de los graduados de la Escuela Profesional de Administración, Universidad Nacional «Santiago Antúnez de Mayolo»; así, sobre la base de los resultados, la mayoría no estuvo trabajando en algún momento durante los dos últimos años del pregrado, por lo que existía una tendencia a la insatisfacción laboral de los titulados con su primer empleo; así también, en relación con este punto, los resultados muestran que el principal motivo por el que trabajaron en pregrado los ahora titulados fue por necesidad económica, la mayoría de encuestados manifestó no haber hecho prácticas en una empresa o institución mientras estudiaba, esto respecto a experiencia profesional y, en cuanto a elementos importantes del empleo, la mayoría indicó que tardó de cinco a más meses en hallar el primer empleo después de titularse, que la categoría profesional de su primer empleo fue de administrativo; la mayoría de encuestados 
respondió que no tenían persona o grupo a su cargo, y que el tipo de contrato para la mayoría fue temporal.

En relación con lo señalado, las bases teóricas van en esa línea, pues se destaca el valor creciente de las prácticas durante los estudios y/o en algunas carreras, como vía de reclutamiento al finalizarlas. Las prácticas tienen un importante valor formativo y una repercusión directa en el currículum, ya que para muchos estudiantes son la primera experiencia profesional en su ámbito, hecho que permite no dejar en blanco el apartado de experiencia laboral. Las prácticas son también un espacio importante de observación del graduado por parte del empleador, quien puede valorar en un contexto real de trabajo si la persona responde al perfil de una posible vacante, si encaja en una cultura de la empresa y qué cualidades y potencialidades tiene. Por último, los servicios de orientación e inserción para los universitarios pueden contribuir también a dar a conocer los candidatos a las empresas y facilitar prácticas de trabajo.

Escalona (2006), señala también que la educación juega un papel económico bastante fuerte en cualquier país y dado el desarrollo de los países, la oferta laboral tiende a caracterizarse por desarrollar actividades encaminadas a bienes y servicios. Se parte de la idea de que la educación superior se debe relacionar con el mercado de trabajo para evitar la ineficiencia en el uso de recursos económicos y sociales, ya que con una educación adecuada al mercado se reduciría el desempleo y apoyaría al crecimiento económico de cualquier país. Por tanto, es necesario establecer la vinculación del sector productivo con la educación profesional. «Es evidente que los niveles de empleo y desempleo dependen de la oferta de mano de obra tanto como de la demanda. La mano de obra plantea cuestiones relativas a la cantidad y la calidad de los trabajadores en busca de empleo» (Psacharapolus, 1989: 77). Por último, desde el punto de vista metodológico, el enfoque de la presente investigación impide generalizar los resultados para otras carreras afines o egresados con otros planes curriculares. Por lo tanto, proponemos que se incluya también en estudios muestras correspondientes a graduados con otros planes curriculares y graduados de otras carreras afines correspondientes a la misma promoción de graduados.

\section{CONCLUSIONES}

El marketing personal influye significativamente en el empleo de los graduados de la Escuela Profesional de Administración de la Universidad Nacional Santiago Antúnez de Mayolo debido a que los titulados que no acuden a cursos de formación sobre búsqueda de empleo o de orientación profesional presentan una tendencia hacia valoraciones de insatisfacción con el empleo en general respecto a su primer trabajo. 
Los factores psicosociales se relacionan directa y significativamente con el nivel de empleo de los graduados de la Escuela Profesional de Administración de la Universidad Nacional Santiago Antúnez de Mayolo, pues la mayoría de titulados que durante los dos últimos años de pregrado solo se dedicaron a estudiar a tiempo completo, sin alternar los estudios con algún trabajo eventual, señalaron valoraciones en grados de insatisfacción laboral.

La experiencia preprofesional se relaciona directa y significativamente con el empleo de los graduados de la Escuela Profesional de Administración de la Universidad Nacional Santiago Antúnez de Mayolo debido a que son bajas las calificaciones de los titulados cuya situación laboral es la de inactivos respecto al grado de acuerdo con cada uno de los 16 elementos del total de siete factores psicosociales (importancia que otorgan al empleo, atribución de las causas del empleo, disponibilidad para el empleo, autovaloración personal y profesional, estilo de búsqueda de empleo, madurez ocupacional y habilidades para la búsqueda de empleo).

\section{REFERENCIAS BIBLIOGRÁFICAS}

Acosta, José. 2006. Marketing personal. Madrid: Esic.

Alles, Martha. 2012. Diccionario de términos de recursos humanos. Buenos Aires: Granica.

Caballero, Gonzalo y Kinsgton, Christopher. 2005. Capital social e instituciones en el proceso de cambio económico. Ekonomiaz. 1-25.

Cáritas Española. 1999. ¿Cómo desarrollar la empleabilidad? Cuadernos para la Inserción Laboral. Madrid: Cáritas Española.

Escalona, Lina. 2006. Formación profesional y mercado laboral: via real hacia la certificación del bibliotecólogo. México D.F.: Universidad Nacional Autónoma de México.

Fernández, Guillén y otros. 2001. La orientación socio-laboral en el itinerario de inserción realizado por Cáritas. España: Universitat Jaume I.

Granovetter, Mark. 1973. The Strength of Weak Ties. en American Journal of Sociology, $78(2), 1371$.

Jiménez, Héctor. 2013. Marketing Personal o el Arte de Vender sus Servicios. (Trabajo de investigación). México D.F.

Psacharapolus, George. 1989. Los lazos entre la educación y el mercado laboral: una perspectiva más amplia. Docencia pos-secundaria, secundaria 17(2), 77. 
Sáenz, Pamela. 2013. Formación técnica empresarial y la inserción laboral de los egresados del Centro Técnico Productivo Teófilo Méndez. Ramos de Huaraz, 2012 (Tesis para optar el grado de Licenciado en Administración). Universidad Nacional Santiago Antúnez de Mayolo, Huaraz.

Torres, Luis. 2011. Propuesta de un plan de marketing para una marca personal, 2011. (Tesis para optar el Grado de Maestro en Marketing Internacional de la Escuela de Postgrado de Marketing Internacional). Buenos Aisres.

Fecha de recepción: 22 de agosto de 2016

Fecha de aceptación: 15 de noviembre de 2016

\section{Correspondencia}

Telmo Loli Poma

pablololi@fatunasam.com 